\title{
Intrinsicality, Independence and Grounding
}

\begin{abstract}
This paper investigates the plausibility of Witmer, Butchard and Trogdon's (2005) proposal to distinguish intrinsic properties from extrinsic ones in terms of independence from accompaniment and grounding. I argue that the proposed criterion is not adequate to determine intrinsicality, since according to it some intuitively extrinsic properties turn out to be intrinsic. I suggest and evaluate two responses: first, one could characterize a conception of independence which is specific to the individual instantiating the property; and second, one could justify two assumptions about properties which entail that counterexample properties of the kinds I present do not exist, most importantly that there are no fundamental properties which are instantiated in an intrinsic fashion by some individuals and an extrinsic fashion by others. Although the latter seems prima facie plausible, I present some potential counterexamples to it from current physical theory. I conclude that the grounding- and independence-based criterion of intrinsicality can be defended from my objections, although the cost of doing so might make it more attractive to characterize intrinsicality in terms of independence, or in terms of grounding, alone.
\end{abstract}

Key Words: Intrinsic and Extrinsic Properties, Grounding, Independence, Loneliness 


\section{Intrinsicality, Independence and Grounding}

Intuitively, we distinguish intrinsic from extrinsic properties; roughly speaking, the properties which a particular has in virtue of the way that it is, and those which require the existence of other entities in order to be instantiated. This distinction has a range of philosophical uses: intrinsicality marks out the properties which are important to duplication; to the causal efficacy which a particular has, and to which supervenient properties it might instantiate (although externalists about the mind would contest precisely that point); a change in the intrinsic properties of a particular is a real change; and a particular's having value in virtue of its intrinsic properties - either ethical or aesthetic - make that particular valuable in itself, rather than because others treat it as valuable.

Until fairly recently, philosophers were content to rely upon an intuitive distinction between intrinsic and extrinsic properties, but a surge of interest in clarifying what exactly this distinction amounts to has led to a corresponding surge in proposals about how it might be drawn. ${ }^{1}$ This paper will investigate the viability of a hybrid criterion of intrinsicality which is based on two key notions: independence - that an intrinsic property is one which could be instantiated by an individual whether or not anything else exists - and grounding. If such a criterion works, its proponents claim, it promises to be both wide in scope-covering a wide range of families of properties - and minimal in the metaphysical commitments it requires. I will argue that, even if we presuppose that there is a coherent notion of grounding, the proposal is inadequate because it classifies some intuitively extrinsic properties as intrinsic.

I will then suggest two responses to the objection I have raised: first, one could strengthen the criterion by formulating it in terms of a revised conception of the independence of a property which is relativized to that property's instantiation by a specific individual; or, second, one could make explicit two assumptions concerning properties, grounding and intrinsicality which would entail that no counterexamples of the type I have presented exist. Although these assumptions have intuitive appeal, I will suggest that there are reasons, based on examples of properties in current physical theory, why one might not be comfortable accepting the most important of these that no fundamental properties are instantiated in an intrinsic fashion by some individuals and in an extrinsic fashion by others. Such misgivings turn out to be all the more important since this

1 Criteria have been suggested by Kim (1982), Lewis (1983a), Langton and Lewis (1998), Francescotti (1999), Witmer, Butchard and Trogdon (2005), Harris (2010), Rosen (2010), Witmer (2014) among others. For a defence of why a strict criterion is required over and above our orienting characterizations of intrinsicality, see Witmer 2014, 1.2 . 
assumption might well be required for either response to work. I conclude that the grounding-based criterion of intrinsicality can be defended but that doing so either involves a greater level of ontological or theoretical commitment to which kinds of properties there are than its proponents intended, or it requires a closer association between grounding and intrinsicality than initially presumed. Thus, it does not have a clear advantage over accounts of intrinsicality based on independence or on grounding alone.

\section{Independence and Naturalness}

I will primarily discuss the account presented by Gene Witmer, William Butchard and Kelly Trogdon (2005) which draws upon an earlier proposal by Rae Langton and David Lewis (1998, 2001). Langton and Lewis noted that it is a necessary condition for a property's being intrinsic is that it is independent of loneliness and accompaniment; that is, a property is intrinsic only if it can be instantiated by a particular whether or not anything else exists. This can be summarized as follows:

Independence: $\quad \mathrm{P}$ is independent of loneliness and accompaniment if and only if

(i) It is possible for a lonely thing to have $\mathrm{P}^{2}$

(ii) It is possible for a lonely thing to lack $\mathrm{P}$

(iii) It is possible for an accompanied (non-lonely) thing to have $\mathrm{P}$

(iv) It is possible for an accompanied thing to lack $\mathrm{P}$

However, although independence is a plausible necessary condition for intrinsicality, it is not a sufficient condition, since there are properties which are independent of accompaniment and nevertheless intuitively extrinsic. For instance, an existential property such as being such that there is a bear can be had by a lonely bear, and an accompanied bear, lacked by a lonely human and an accompanied human; as can disjunctive properties such as being spherical and lonely or nonspherical and accompanied.

In order to make their criterion adequate, Langton and Lewis account for such properties by ruling out disjunctive properties: a basic intrinsic property is independent, and neither disjunctive nor the negation of a disjunction. But to do that, they must also distinguish spurious disjunctive properties from genuine ones: it is, for instance, possible to make any property $\mathrm{P}$ into a disjunction, since being $P$ is necessarily coextensive with being either $P$ and $Q$ or $P$ and not $Q$. Langton and

2 A (concrete particular) thing is lonely in world iff it is the only thing which exists in that world. 
Lewis need to exclude such artificial disjunctive properties in order to retain a core set of properties which are not disjunctive, and they do so by recourse to the primitive notion that some properties are more natural than others. Thus, an apparently disjunctive property does not count as genuinely disjunctive if its disjuncts are less natural than the disjunction as a whole; or, conversely, a genuinely disjunctive property is one such that its disjuncts are more natural than it is. Langton and Lewis's additional constraint is sufficient since it excludes the overtly disjunctive properties which satisfy the independence criterion, and also excludes existential properties, such as being such that there is a bear, as long as it is permissible to regard such a property as a disguised disjunction of the form being either a bear or a non-bear accompanied by a bear.

One might be troubled by at least two points about Langton and Lewis's account of intrinsicality ${ }^{3}$ : first, one might worry about the plausibility of their supposition that existential properties can be analysed as being disguised disjunctions in order that they turn out to be extrinsic properties according to the Langton-Lewis definition (Marshall and Parsons 2001); and second, one might question the introduction of property naturalness, its intelligibility and why greater naturalness would be associated with intrinsicality as Langton and Lewis suggest. The LangtonLewis criterion works - if it works at all-by presupposing a hierarchy of properties and although Langton and Lewis are quite flexible about what naturalness involves, it is nevertheless a significant commitment to ontology or ideology which one might want to avoid in the course of distinguishing intrinsic from extrinsic properties. ${ }^{4}(1998,119-20)$

Perhaps the most plausible explanation of why greater naturalness should be a guide to intrinsicality is suggested by Lewis in his previous work in which he makes a specific ontological commitment to the existence of an elite set of sparse, perfectly natural properties. (1983b, 192-4) Such properties are, by definition, fundamental, intrinsic, and sharing of them makes for duplication and resemblance; they carve nature at the joints and determine all the similarity and difference in the world, with naturalness of other properties being a matter of degree according to their closeness in the hierarchy to the perfectly natural ones. The naturalness of a property increases the more closely connected it is to intrinsic perfectly natural properties, which makes it clear why greater naturalness and intrinsicality should go together. However, although Lewis remained convinced by this ontological commitment to perfectly natural properties and the account of intrinsicality he

3 It is not my primary objective to consider objections to the Langton-Lewis account here. See Marshall and Parsons 2001, Sider 2001 and Witmer, Butchard and Trogdon (2005, 328-331).

4 Whether the commitment to a hierarchy of property naturalness is ontological or ideological depends upon what one thinks properties are and what makes it the case that one property is more natural than another; in particular, whether relative naturalness is an objective matter or a question of which properties are more central to our best theories (presuming, for a moment, that these two options do not coincide). For a representative of the former view, see Lewis 1983b; for the latter, see Taylor 1993. 
based upon $\mathrm{it}^{5}$, he appreciated that accepting the objective existence of sparse perfectly natural properties should not be mandatory in order to distinguish intrinsic from extrinsic properties, and the Langton-Lewis appeal to a broader conception of naturalness is an attempt to avoid such a requirement. However, once one broadens the conception of naturalness and it is no longer characterized as a connection to properties which are essentially intrinsic, it is no longer clear why greater naturalness should be a guide to intrinsicality at all.

\section{Independence and Grounding}

Witmer, Butchard and Trogdon (henceforth WBT) adapt the Langton-Lewis account in order to remove the potentially problematic reliance on naturalness. Their proposal characterizes the intrinsicality of a property in terms of its being independent of loneliness and accompaniment and also its being grounded in the individual which has it by other properties had by that individual which are also independent of accompaniment. Like naturalness, grounding is a metaphysical notion $^{6}$ and so the account is not purely logical, but WBT argue that grounding is less opaque and obscure than naturalness, and thus that their account has greater clarity. Although it was originally named 'the Strong Independence Definition' $(2005,331)$, this account is more commonly known by the initials of its proponents as the WBT Grounding account.

The proposal involves two conditions and we can characterize it as follows:

\section{WBT Grounding:}

A) Property $\mathrm{P}$ is had by individual $\mathrm{b}$ in an intrinsic fashion iff

(a) $\mathrm{P}$ is independent of loneliness and accompaniment;

(b) If $\mathrm{P}$ is instantiated by $\mathrm{b}$ at least partially in virtue of other properties of $\mathrm{b}$, each of these is also independent of loneliness and accompaniment.

B) Property $\mathrm{P}$ is intrinsic iff any possible individual $\mathrm{x}$ which has $\mathrm{P}$ has $\mathrm{P}$ in an intrinsic fashion.

There are three preliminary points to note about this definition. First, that 'having a property in an intrinsic fashion' is a relativized notion in that a property $\mathrm{P}$ may be instantiated in an intrinsic fashion by some individuals and not by others which nevertheless instantiate it. For instance, the property of being a cube, or non-spherical and accompanied is had in an intrinsic fashion by cubes,

5 I will not be concerned with Lewis's original account in what follows (1983b), which maintains that a property P is intrinsic if and only if for any two possible duplicates, either both have $\mathrm{P}$ or both lack it. Duplication is then analysed in terms of the sharing of perfectly natural properties.

6 As with the broader conception of naturalness, one might suggest that grounding can be ideological as well as ontological. (See Thompson (forthcoming).) However, this does not appear to be the way in which WBT intend grounding to be understood and so I will treat grounding as an objectively 'real' relation in what follows. 
while it is not had in an intrinsic fashion by non-spherical things which are accompanied by some other contingent individual, when the property is instantiated by an accompanied pentagon (say). Similarly, the property of being such that a bear exists is instantiated in an intrinsic fashion by any bear, and a non-intrinsic fashion by non-bears who coexist with bears. If this relativization is considered to be problematic, then the proposal is not viable and will have to be abandoned. (Hawthorne 2001, 400 n1; WBT 2005, 333-4.) But it is not clear why it should be a problem, since the relativization simply amounts to accepting that some properties can be locally intrinsic which are not globally so; that is, some properties can be instantiated in an intrinsic fashion by some particulars and not by others. Condition (B) adds the requirement for intrinsicality that only those properties which are locally intrinsic in all possible individuals which instantiate them are intrinsic. There does not seem to be a viable objection here.

Second, the definition may adversely affect the viability of the criterion of property identity based upon necessary coextension because on this definition, necessarily coextensive properties can differ in whether they are intrinsic or not. (WBT 2005, 334) For example, consider the intrinsic property $\mathrm{Q}$ and the property being $Q$ and accompanied or $Q$ and lonely; clearly, any individual which has $\mathrm{Q}$ is also either lonely or accompanied, and so it also instantiates the property of being $Q$ and accompanied or $Q$ and lonely, but while $\mathrm{Q}$ is intrinsic, the latter property is not. This may lead one to question whether the identity criterion for properties needs to permit finer-grained distinctions between properties — so that being $Q$ is distinct from being $Q$ and accompanied or $Q$ and lonely — which suggests the plausibility of a hyperintensional criterion. Or, it may be taken to imply that the properties of being intrinsic and being extrinsic are not directly possessed by properties - contrary to what we have been assuming — but are instantiated by finer-grained entities such as predicates, meanings or Fregean concepts instead. In the latter case, whether a property is intrinsic or not depends upon how it is described, upon the predicate used to pick it out. WBT consider this dilemma to be an acceptable consequence of their proposal $(2005,334)$, but it is not clear that either option is a satisfactory one. I will not explore these options further however, since I think there is a more serious problem with their proposal as it stands. ${ }^{7}$

Third, one might raise some serious questions about the use of grounding. What does 'in virtue of' mean? Unless we can give an account of grounding, or adequately explain what the 'in virtue of' locution means in this context, then WBT Grounding has not delivered the advantage over Langton and Lewis's naturalness account which its proponents promised. It is, on the worst assessment of it, either obscure, or circular, or it does not define 'intrinsic property' as we usually

7 The relationship between grounding-based accounts of intrinsicality and property identity is considered in greater detail in Marshall 2015. 
intend such a notion. It would be guilty of obscurity were our understanding of 'in virtue of' no clearer than our understanding of property naturalness. However, WBT reject this charge because the 'in virtue of' locution is widespread through a swathe of philosophical discourse while property naturalness is a specialized notion, invoked as a primitive in Lewis's theory and then broadened by Langton and Lewis. But this may be too quick a response, since the popularity and entrenchment of a phrase in philosophy does not automatically yield transparency of meaning, and furthermore, its use might not be unequivocal in all philosophical contexts; just because philosophers use a phrase does not entail that they know what it means, and certainly does not imply that they are all describing the same phenomenon. On the other hand, Lewis introduces naturalness for the specific task of capturing the relation between a property and more fundamental properties in terms of boolean operations, and although it is a novel term, it may claim to be just as clear as 'in virtue of'. (1983b, 193) One might think that the same defence is not available for Lewis and Langton's more flexible version of naturalness in their account of intrinsicality (1998, 119-20); but one could respond that each specific version could be made just as clear.

The problem of circularity would arise if, in the context of defining intrinsic properties, 'in virtue of' could only be understood by presupposing a prior notion of intrinsicality. WBT deny that their account is directly circular in this way $(2005,337-8)$; however, without a careful explication of grounding - which goes beyond the scope of this paper - it is difficult to assess whether they are right. Nevertheless, we can take an optimistic approach and presume that a suitable definition of grounding is available (or that definitions are available, at least one of which is adequate to meet our needs ${ }^{8}$ ), or else we could treat grounding as a primitive metaphysical relation and define intrinsicality in terms of it. So I will not treat the challenge of explicating grounding as a fatal objection to WBT's criterion of intrinsicality, although the need for a clearer account of grounding will become more pressing in Section 4.

\section{Problems with the Sufficiency of WBT Grounding}

The main weakness of WBT Grounding as it stands concerns the sufficiency of the criterion: there are properties which satisfy conditions (A) and (B) and yet are intuitively extrinsic. I will first present two objections, concerning parts (a) and (b) respectively of condition (A), and briefly consider WBT's pre-emptive response to the second which attempts to explain away such counterexample properties. Their response is, I argue, unconvincing. I will then suggest two

8 See Trogdon 2013, Schaffer 2009 who consider grounding to be univocal, and Hofweber 2009 and Fine 2012 who think that there are different kinds of grounding relations; Bennett 2017 argues that there is a resemblance class of relations which are not exactly similar. 
alternative responses: one which attempts to strengthen the criterion in such a way that the counterexamples do not arise, and a second which aims to clarify the assumptions which WBT are implicitly making (or, which they appear to be making) in order to sustain both their original position and their response to objections of the kind I have raised.

\subsection{Two Objections}

The first objection concerns part (a) of condition (A), which, in order to avoid counterexamples, requires an implicit assumption that there are no ungrounded locally intrinsic properties; that is, there are no ungrounded properties which are both independent from loneliness and accompaniment ${ }^{9}$ and instantiated by some individuals in an intrinsic fashion and by other individuals others in a non-intrinsic fashion. If there were such properties, they would satisfy condition (A) which requires only that an ungrounded property is independent for it to be classed as being instantiated by an individual $b$ in an intrinsic fashion, since condition (b) of (A) would not apply.

For instance, let us assume for a moment that the locally intrinsic property being such that there is a bear is an ungrounded property. (I have chosen the example for simplicity, not because of its plausibility as an ungrounded property and the assumption will quickly be dropped.) Given that bears exist, this is a property which I instantiate, so I will be suitable as the individual $b$ in the example. ${ }^{10}$ As noted above, being such that there is a bear is independent so it passes part (a) of condition (A) and since, by hypothesis, it is ungrounded, (b) would not apply to it. Thus, (A) is satisfied and according to WBT Grounding I instantiate being such that there is a bear in an intrinsic fashion. Furthermore, this example generalises and so any other non-bears who coexist with bears also instantiate being such that there is a bear an intrinsic fashion. Since any possible individual which has this property has it in an intrinsic fashion, condition (B) is satisfied too and so being such that there is a bear turns out to be intrinsic according to WBT Grounding. This result would obtain in the case of any property which is independent and ungrounded, and so if any locally intrinsic properties meet this requirement, they will wrongly count as globally intrinsic according to WBT Grounding.

The second difficulty with WBT Grounding concerns part (b) of (A). There is another class of counterexamples which are intuitively had by an individual $b$ in an extrinsic fashion and which nevertheless satisfy (A), the criterion for their being had by $b$ in an intrinsic fashion. Given the

9 I will abbreviate this to 'independent of accompaniment' or 'independence' in what follows.

10 I am not a bear. 
independence of constraints (A) and (B), at least some such properties may then be able to satisfy (B) as well, with the result that some intuitively extrinsic properties pass WBT's test of intrinsicality.

According to (A), for a property $\mathrm{P}$ to be instantiated in an intrinsic fashion in a particular $\mathrm{b}$, the property $\mathrm{P}$ and each property upon which $\mathrm{P}$ (at least partially) depends must be independent. So, if $b$ has $\mathrm{P}$ in virtue of having $\mathrm{Q}, \mathrm{Q}$ must be independent. The following objection concerns properties such as $\mathrm{Q}$, upon which the instantiation of property $\mathrm{P}$ (whose intrinsicality is at issue) depends, and claims that requiring the independence of such grounding properties is not sufficient to ensure that $\mathrm{P}$ is instantiated in an intrinsic fashion.

Let us suppose that the following claims are true:

(i) $\mathrm{P}$ is independent

(ii) Q is independent

(iii) $\mathrm{b}$ has $\mathrm{P}$

(iv) b has Q

(v) The fact that $\mathrm{b}$ has $\mathrm{P}$ is partially (possibly fully) grounded by the fact that $\mathrm{b}$ has $\mathrm{Q} .{ }^{11}$

Let us also presuppose that if the fact that $\mathrm{b}$ has $\mathrm{P}$ is only partially grounded by the fact that $\mathrm{b}$ has $\mathrm{Q}$, then all other grounding properties of $\mathrm{P}$ are also independent, since if they are not, $\mathrm{P}$ will fail to satisfy (A) on account of them, making P extrinsic. (The latter case of non-independent grounding properties is not problematic for WBT Grounding and I mention it simply to rule it out.) There will be reason to discuss this presupposition in greater detail later (see 4.2).

As noted in Section 1, a property can be independent and yet extrinsic. When such properties are the principal property under consideration-property P in the WBT Grounding criterion - they are prevented from counting as intrinsic because they do not satisfy condition (B): that is, they are not had in an intrinsic fashion by every possible individual which instantiates them. (I will, for the moment, only consider cases where the instantiation of $\mathrm{P}$ is grounded by the instantiation of other properties, and thus presume that my first objection does not apply.) However, WBT Grounding does not exclude such independent and extrinsic properties from being among those which ground other properties. What if $\mathrm{Q}$ is such an independent and yet extrinsic property? Premises (i)-(v) could be true, so that $\mathrm{P}$ satisfies criterion (A) to be instantiated by $\mathrm{b}$ in an intrinsic fashion, even though $\mathrm{P}$ is grounded by property $\mathrm{Q}$ which is extrinsic.

11 (v) presupposes that facts are the relata of the grounding relation, although I will not argue for this point. One could recast premise (v) as a relation between property instantiations or between 'modes' of b (b's having Q partially (or possibly fully) grounds b's having P) without affecting the argument. For the general case, WBT write about properties grounding other properties and so I will also use that locution in what follows (for instance, 2005, 340). 
Moreover, in such cases, $\mathrm{P}$ could also satisfy condition (B) of being instantiated by any possible individual in an intrinsic fashion. (There seems to be no obvious reason why satisfying (B) should not be possible, at least in some cases, since the current fault with (A) as it stands makes being instantiated by an individual in an intrinsic fashion easier to achieve than it should be.) Thus, it is possible that a property which is intuitively extrinsic, because it is grounded by an extrinsic property or properties, counts as intrinsic on the WBT Grounding criterion.

These two objections show that, as it is presented, WBT Grounding allows both ungrounded, independent extrinsic properties and properties grounded by independent extrinsic properties to satisfy (A). Properties such as being such that there is a bear, or having the mass of an electron, or being the size of Wales would all, were they to be ungrounded, be properties which satisfied the WBT Grounding criterion for being intrinsic and yet they are extrinsic; thus, they fail according to the first objection. (In Section 4.2, I will argue that there are some more familiar examples of ungrounded properties which may plausibly be thought of as being locally intrinsic properties, including mass and spin. If I am right, these too would be extrinsic and yet turn out be intrinsic according to WBT Grounding.) The second objection would apply to any properties which are grounded or partially grounded by properties such as these, including having a genuine fear of bears, or having the mass of a hydrogen atom, for example. Such intuitively extrinsic properties turn out to be intrinsic according to the WBT Grounding criterion and so the criterion is inadequate.

\subsection{The no-response response}

One might think that there is no need to respond to these objections. Perhaps we could just exclude the problematic properties upon which my objections are based from being covered by the criterion; in particular, the locally intrinsic properties which can be instantiated in an intrinsic fashion by some individuals and in an extrinsic fashion by others. After all, it is not unusual to exclude families of properties from being covered by a potential criterion of intrinsicality, such as haecceistic properties which can be instantiated by one particular (such as being Obama), ${ }^{12}$ or necessary properties which are indiscriminately instantiated by everything (such as being self-identical or being such that 37 exists). However, it is not clear that it would be possible to isolate locally intrinsic properties as a group without gathering such properties on an ad hoc basis. Furthermore, even if this were possible, saving WBT Grounding by restricting the range of properties to which it applies would leave it poorer as a result: the criterion would lose generality and we would forego the means to judge that such properties are globally extrinsic as they so obviously seem to be. A 
response to the objections which allowed WBT Grounding to correctly classify locally intrinsic properties would be preferable to narrowing the scope of the criterion so that it does not apply to them.

\subsection{WBT's Response}

WBT are aware that one may question the sufficiency of their criterion and they do attempt to address counterexamples to condition (A). (2005, 345-7) However, although their discussion of this point provides a useful pointer towards their way of thinking about both grounding and intrinsicality, their defence is not a strong one: they do not address counterexamples of the type suggested in the first objection of ungrounded, independent and yet extrinsic properties; nor do they give compelling assurances that those in the second objection can be ruled out. Rather, their response is more of an assertion that such counterexamples should not arise:

'If our definition succeeds, for each [counterexample property] we should be able to

find some possible case in which an individual has the property by virtue of having some other property that is not independent of accompaniment.' $(2005,346)$

Their claim is correct, of course. But it will hardly serve to convince someone who is not yet assured that WBT Grounding will succeed; that is, that WBT Grounding is the correct way of characterizing intrinsicality and so the counterexamples to it are merely apparent. What is required is some justification for a general principle that any property which is instantiated by an individual in an extrinsic fashion is at least partially grounded by a property of that individual which is not independent.

WBT attempt to demonstrate that there are such non-independent grounding properties for two counterexamples to sufficiency, being such that there is a cube (Marshall and Parsons 2001, 349) and being such that there is something to which one is attending (Hawthorne 2001, 399). Both properties are independent: a cube would instantiate the former in an intrinsic fashion while a noncube accompanied by a cube would not; and the latter would be instantiated in an intrinsic fashion by someone attending to something intrinsic to herself - to her left knee, or to her most recent dream - while it would be had in an extrinsic fashion by someone attending to something else. Moreover, when these properties are instantiated in an extrinsic fashion, their grounding properties are not guaranteed to lack independence: a non-cube might have the former property in an extrinsic fashion in virtue of being accompanied by a cube, and one might have the latter in an extrinsic fashion simply in virtue of attending to something, and both of these properties are independent. (WBT 2005, 346) However, were the respective grounding properties to include being 
accompanied by a wholly distinct cube, and attending to something wholly distinct from oneself, then being such that there is a cube and being such that there is something to which one is attending would be at least partially grounded by properties which failed to be independent and would turn out to be extrinsic as required. WBT have to motivate the claim that the latter pair of grounding properties are the relevant ones rather than those which do not explicitly involve distinctness from the instantiating individual. But the fact that we have to make a choice between grounding properties to deal with counterexamples is cause for concern. Why should we accept the property which is not independent of accompaniment as the relevant grounding property rather than one which is independent? To choose the former rather than the latter simply to defend WBT Grounding would be ad hoc. Furthermore, one might also be worried about the mention of distinctness from the instantiating particular in the description of the grounding properties themselves. Is it possible, for instance, to clarify what counts as an individual being wholly distinct from another without presupposing that they share no intrinsic properties?

WBT's justification for their choice of non-independent grounding properties when independent ones are plausible is complicated by the fact that they have previously argued for the irrelevance of certain grounding properties in some cases $(2005,341)$; in particular, in cases where extrinsic properties could be considered to ground intuitively intrinsic properties. For instance, they point out that an accompanied sphere instantiates being a sphere, but one might consider that this property is grounded by the individual sphere's being accompanied and being a sphere and accompanied or a non-sphere and lonely, which would make being a sphere count as being instantiated in an extrinsic fashion. The set of properties which are jointly sufficient to ground being a sphere might include non-independent properties as members when independent ones would do. Although I agree with WBT that there is 'no intuitive pull' towards regarding these extrinsic grounding properties as being the relevant ones $(2005,341)$, a heavy dose of intuition appears to be required in addition to WBT Grounding to sort 'legitimate' grounding properties from 'spurious' ones and this is cause for concern. If intuition intrudes again in order to rectify counterexamples to sufficiency, but in this case it does so by including non-independent properties among the grounding properties (such as in the cases in the previous paragraph) when a set of independent ones would be jointly sufficient, then this counts further against the plausibility of WBT Grounding. What is required is a criterion which can stand alone, rather than one which is propped up by ruling out the problem cases in what threatens to be an ad hoc way.

It would help if WBT could tell us why we should share their intuitions on these matters and in the case of the sufficiency counterexamples they do so $(2005,346)$ : the way in which an individual has a property in an intrinsic fashion is different from the way another individual 
instantiates that same property. For instance, the way in which being such that there is a cube is instantiated by a cube is different, WBT urge, to the way in which it is instantiated by an individual accompanied by a cube. Furthermore, the difference is revealed in the property having different grounding properties. $(2005,347)$

Although it is intuitively attractive, this appeal to the ways in which a property is instantiated is not particularly helpful to justify judgments about which grounding properties are relevant to the instantiation of a property in an intrinsic (or an extrinsic) fashion and which are not. The reason for this is that there are often many different 'ways' in which a property might be instantiated which do not indicate differences which are relevant to whether a property is had in an intrinsic fashion or not. This plenitude of ways can be illustrated if we consider properties which are variably realized; that is, properties which can be instantiated in virtue of different sufficient sets of grounding properties in different individuals (or in the same individual at different times). Such a variably realized property, such as being hungry or thinking about one's last dream, might be instantiated in many different ways corresponding to the different sets of grounding properties in different individuals (or the same individual at different times). Moreover, the difference between these different ways can be explained by different instantiations of the property having different grounding properties, just as WBT suggest that the different ways in which a property is instantiated in an intrinsic fashion and an extrinsic fashion can be distinguished. $(2005,347)$ But such a variably realized property might always be instantiated in an intrinsic fashion, and thus be globally intrinsic, despite the intuitive differences in the ways in which different individuals instantiate it. ${ }^{13}$ These different ways are not relevant to whether an individual has the property in an intrinsic fashion or not, which prompts the question of whether we can distinguish ways which are relevant to making the intrinsic/extrinsic distinction from those which are not. If we attempt to clarify the matter by appealing to the fact that in the intrinsic case the individual $\mathrm{b}$ has property $\mathrm{P}$ in virtue of how $\mathrm{b}$ itself is, while another individual $\mathrm{c}$ instantiates $\mathrm{P}$ in virtue of entities distinct from itself, we are running the risk of getting caught in a tight conceptual circle: what makes b how it is but the properties which $\mathrm{b}$ has in an intrinsic fashion? Why are the properties which $\mathrm{b}$ has in an extrinsic fashion irrelevant to how $b$ itself is? It seems that the relevant difference in ways of instantiating a property which we require just is different individuals' instantiating a specific property in an intrinsic or in an extrinsic fashion. So far, appealing to the different ways in which a property can be instantiated in order to eliminate the counterexamples to the sufficiency of WBT Grounding has not permitted much explanatory progress. I will return to it when I consider my own suggestions.

13 Similarly, a property might be instantiated in different ways — in virtue of different grounding properties — and always be instantiated in an extrinsic fashion. There will also be mixed cases (as illustrated by Hawthorne's example above). 
WBT's response to the counterexamples would be improved if they were either able strengthen the conditions which apply to grounding properties in condition (A), and thereby preclude properties which are intuitively had in an extrinsic fashion from qualifying as being instantiated in an intrinsic fashion, or to articulate and justify stronger, general reasons why we should not expect counterexamples to their criterion to arise. I will consider these two responses in turn.

\section{Can the grounding-based criterion of intrinsicality be improved?}

\subsection{Strategy 1: Strengthening WBT Grounding by relativizing Independence.}

If we are to strengthen the requirement on grounding properties in condition (A), it will be useful to clarify what is intuitively required of grounding properties in order to guarantee that the properties which they ground are instantiated in an intrinsic fashion by an individual. Intuitively, the crucial factor in whether $\mathrm{P}$ is instantiated by $\mathrm{b}$ in an intrinsic fashion is not simply whether grounding property $\mathrm{Q}$ is intrinsic or extrinsic in general, but whether $\mathrm{Q}$ is instantiated $b y b$ in an intrinsic or an extrinsic fashion. ${ }^{14}$ For instance, if an independent property $\mathrm{S}$ which I instantiate is grounded by my instantiating the property of being such that there is a bear, that property $\mathrm{S}$ is intuitively instantiated by me in an extrinsic fashion, even though $\mathrm{S}$ satisfies (A) and so according to WBT Grounding, $\mathrm{S}$ is had by me in an intrinsic fashion. ${ }^{15}$ On the other hand, if independent $\mathrm{S}$ is instantiated by an individual bear and is grounded by the same property of being such that there is a bear, it seems correct to say that $\mathrm{S}$ is instantiated by the bear in an intrinsic fashion because the grounding property of S-being such that there is a bear-is had by the bear in an intrinsic fashion.

Perhaps one could capture the requirement that the grounding properties of a property had in an intrinsic fashion by an individual must themselves be instantiated by that individual in an intrinsic fashion. One might do so by introducing a conception of independence which is relativized to the individual $\mathrm{b}$ instantiating the property $\mathrm{P}$, such that a property is independent when instantiated by an individual $b$. Let us call this Independence* and define it as follows:

14 WBT agree that what matters is which grounding properties each individual has, rather than which properties ground $\mathrm{P}$ in general. 'It makes no sense to say that $\mathrm{P}$ is had by virtue of $\mathrm{Q}$, simpliciter; we must relativize the question to individuals and ask, in the case of any individual $\mathrm{x}$ with $\mathrm{P}$, what is it about $\mathrm{x}$ that makes it the case that $\mathrm{x}$ has the target property P?' $(2005,332)$

15 WBT would agree with my assessment of S as being extrinsic, since they endorse the following principle (2005, 344): 'For any individual $\mathrm{x}$ and property $\mathrm{P}$, if $\mathrm{x}$ has $\mathrm{P}$ in virtue of some property not itself had in an intrinsic fashion, then $\mathrm{x}$ does not have $\mathrm{P}$ in an intrinsic fashion.' 
Independence*: $\mathrm{P}$ is independent* of loneliness and accompaniment when instantiated by individual $\mathrm{b}$ if and only if

(i) It is possible for $b$ to be lonely and to have $\mathrm{P}^{16}$

(ii) It is possible for $b$ to be accompanied and to have $\mathrm{P}^{17}$

Independence* concerns the relationship between a particular and a property, unlike the more general conception of independence which concerns the relationship between a property and any individual which could instantiate it.

The revised version of condition (A) using independence* would be as follows:

A2) Property $\mathrm{P}$ is had by individual $\mathrm{b}$ in an intrinsic fashion iff

(a) $\mathrm{P}$ is independent of loneliness and accompaniment when instantiated by $\mathrm{b}$ ( $\mathrm{P}$ is independent* when instantiated by $\mathrm{b}$ );

(b) If $\mathrm{P}$ is instantiated by $\mathrm{b}$ at least partially in virtue of other properties of $\mathrm{b}$, each of these is also independent* of loneliness and accompaniment.

Because condition (a) in (A2) requires P to be independent*, the resulting criterion yields results for ungrounded properties in line with our intuitions again and my first objection to WBT Grounding would be refuted. An individual bear would instantiate being such that there is a bear ${ }^{18}$ independently* because the bear instantiates that property whether accompanied or not and so would count as instantiating the property in an intrinsic fashion, while I (in common with other nonbears) would not instantiate being such that there is a bear independently*, and so I would instantiate the property in a non-intrinsic fashion. The property would then no longer satisfy (B): WBT Grounding would count it as extrinsic as required.

Furthermore, the counterexamples used in the second objection to part (b) of condition (A) would also be excluded. If each and every grounding property of $\mathrm{P}$ is required to be independent*, then each property can be instantiated $b y b$ whether or not anything distinct from $\mathrm{b}$ exists, and so the problem cases of independent properties which are nevertheless had by $\mathrm{b}$ in a non-intrinsic fashion will not arise.

The introduction of independence* rules out all the counterexamples to WBT's condition

16 Or else, b's counterparts could do so, if transworld identity of particulars is prohibited.

17 Given that $\mathrm{P}$ might be an essential property of $\mathrm{b}$, it seems unwise to require that $\mathrm{b}$ could lack $\mathrm{P}$ when either lonely or accompanied.

18 Again on the rather implausible assumption that this property is ungrounded. 
(A) and furthermore (A2) appears to put greater conceptual distance between being had in an intrinsic fashion and the conditions required for it than did WBT's talk of the different ways in which a property could be instantiated. However, one might nevertheless be concerned that this conceptual distance is just apparent and wish to investigate independence* more thoroughly: what makes it the case that a particular $\mathrm{b}$ instantiates a certain property $\mathrm{P}$ independently*?

I will not pause to investigate independence* in great detail. ${ }^{19}$ In the worst case scenario, we are again trapped in a tight conceptual circle: the conditions required for independence* obtain solely in virtue of how b is, but that amounts to saying 'how b is intrinsically', or in virtue of the properties which $\mathrm{b}$ has in an intrinsic fashion. Nevertheless, the strengthened version of WBT Grounding can survive even if independence* and being instantiated in an intrinsic fashion cannot be conceptually prised apart (or else if they cannot be conceptually prised apart very far). The resulting condition (A) of the criterion would look something like this, with (B) remaining the same as in previous versions:

\section{A3) Property $\mathrm{P}$ is had by individual $\mathrm{b}$ in an intrinsic fashion iff}

(a) $\mathrm{P}$ is independent of loneliness and accompaniment;

(b) If $\mathrm{P}$ is instantiated by $\mathrm{b}$ at least partially in virtue of other properties of $\mathrm{b}$, each of these is also had in an intrinsic fashion.

It will be noted immediately that 'instantiated in an intrinsic fashion' cannot be used in part (a) of condition (A3) as that would make the criterion circular. Nor can 'independence*' remain in (a), as in (A2), if this amounts to being instantiated in an intrinsic fashion as we are currently pessimistically presuming. Thus, in order to avoid the first objection that $\mathrm{P}$ might be ungrounded, independent and extrinsic, an assumption will be required that there are no such properties. With such an assumption in place, we would have a criterion which determines whether a property $\mathrm{P}$ is had in an intrinsic fashion on the basis of whether P's grounding properties are also had in an intrinsic fashion. Ultimately, any property P's being instantiated in an intrinsic fashion requires either that $\mathrm{P}$ be grounded by fundamental ${ }^{20}$, intrinsic properties or, if nature is infinitely complex and there is no fundamental layer, ${ }^{21}$ that all P's (infinitely many) grounding properties to be

19 The question of what makes it true that a property is instantiated independently* by a particular b requires consideration of what makes the two modal requirements of Independence* true, whether these ultimately depend upon the nature of $b$, and whether the nature of $b$ can be determined without recourse to the properties which $b$ has in an intrinsic fashion. A general discussion of the alternative accounts one might give of this goes beyond the scope of this paper and so I will settle for a discussion of the worst case scenario in which independence* cannot be understood without a prior understanding of a property's being instantiated in an intrinsic fashion.

20 I will treat 'fundamental' and 'ungrounded' as synonyms.

21 See, for example, Schaffer 2003, 505-6. 
instantiated in an intrinsic fashion.

If independence* cannot be understood except as a property's being instantiated in an intrinsic fashion, we will be forced to presuppose that all ungrounded, independent properties are intrinsic. In view of this, one might consider a second defence of the original criterion using this assumption and another one, which together would give us reason to believe that counterexample properties to WBT Grounding of the type I have suggested do not exist.

\subsection{Strategy 2: Adding constraints to WBT Grounding}

To make the second strategy plausible, one needs to justify the following two claims:

(1): There are no ungrounded, independent, extrinsic properties.

(2): Any property which is instantiated in an extrinsic fashion is at least partially grounded by a property which is not independent.

If (1) is true, it alone will neutralise the first objection which highlighted the fact that all ungrounded, independent properties satisfy condition (A), regardless of whether such properties are independent because they are globally intrinsic or because they are only locally intrinsic (and thus extrinsic). Because ungrounded, independent, locally intrinsic properties satisfy (A) for all individuals, even those individuals which intuitively instantiate them in an extrinsic fashion, these properties thereby satisfy (B) and incorrectly count as intrinsic properties according to WBT Grounding. However, if there are no such ungrounded, independent and yet extrinsic properties, then the problem is solved: no such counterexamples can arise.

Moreover, if (1) is true, we have a choice of either strategy: (1) can work alongside independence* if required; or (1) can be combined with (2) to exclude all the counterexamples presented in this paper. The latter option would allow one to avoid commitment to independence* and the potential problems associated with how we should understand it, but it requires that both (1) and (2) be true.

Why should one be committed to the truth of (1)? It is difficult to justify such basic claims about the nature of any category of entities, and one might feel entitled to adopt (1) as a primitive assumption, but I will briefly consider the plausibility of doing so. Several accounts of properties ${ }^{22}$

22 These include both accounts which treat properties as categorical and as essentially dispositional, as well as mixed theories. The differences between these accounts are not relevant to the current discussion. 
presuppose a stronger claim that all fundamental properties are intrinsic (Lewis 1986b, 178; Molnar 2003, 103-7; Mumford 2006, 479-80), which would entail (1). ((1) does not exclude fundamental extrinsic properties, only those which are extrinsic but can sometimes be instantiated in an intrinsic fashion.) Assuming that ungrounded properties are inherently intrinsic seems quite plausible in the context of a general theory of properties. However, in the context of trying to define intrinsicality, it seems presumptive to make such a presupposition without further investigation. Moreover, WBT were eager to avoid any rich and specific ontological commitments in the course of formulating their criterion, such as that they attribute to Langton and Lewis's use of naturalness. However, if I am right, WBT Grounding does not work without (1) which requires that there cannot be any ungrounded properties which can be instantiated by some individuals in an intrinsic fashion and others in an extrinsic one. Since WBT Grounding requires such an assumption, it would be preferable to provide some justification for it.

One might protest that (1) is nigh-on self-evident. But, aside from simply reasserting (1) or asserting that all fundamental properties are intrinsic which entails (1), it is difficult to say why that is the case. A better way to approach the problem might be to ask whether extrinsic fundamental properties are possible, since if there are none, locally intrinsic fundamental properties will also be ruled out. Furthermore, in investigating this matter, we may encounter some properties which are locally intrinsic cases: fundamental properties which can be instantiated by some individuals in an intrinsic fashion and others in an extrinsic fashion, and thus examples of fundamental, independent and yet extrinsic properties. Such properties would act as counterexamples to the truth of (1).

Bauer (2011) has recently suggested that mass, which is a paradigmatically fundamental property, is nevertheless extrinsic because a particle's having the mass it does depends upon the existence of the Higgs Field. ${ }^{23}$ This claim about mass is, especially in the present context, an interesting thesis given that mass is just the kind of property usually considered to be fundamental and intrinsic. However, strictly speaking (as Bauer admits 2011, 93), the existence of the Higgs Field is only relevant to a particle's inertial mass and not obviously to its gravitational mass, which raises the question of how, if one accepts this account, inertial mass relates to gravitational mass. (Furthermore, gravitational mass has two aspects, passive and active: the capacity to be acted on by a gravitational field, and the capacity to generate a gravitational field, respectively.) One can, as Bauer does, cite the physical equivalence of the two varieties of mass which is based on Einstein's equivalence principle that there is no difference between the force felt due to accelerated motion and due to a gravitational field. $(2011,93)$ Thus, he argues that at least passive gravitational mass

23 Crucially, Bauer himself does not think that inertial mass is fundamental however, for reasons which I will address shortly. He does however allow that there may be extrinsic fundamental properties. $(2011,95)$ 
should be aligned with inertial mass as extrinsic. Or else one might accept what Bauer says about the extrinsicality of inertial mass while maintaining that gravitational mass is intrinsic. Which of these options one chooses is not important for my argument, and so presuming that one accepts Bauer's conclusion about the extrinsicality of both inertial mass and passive gravitational mass, one might either take this to indicate that intrinsic active gravitational mass is a distinct property from both passive gravitational mass and inertial mass; or, one might maintain a unified account of mass and accept that mass can be instantiated in both an extrinsic and an intrinsic fashion. If one takes the latter option and also considers mass to be a fundamental property ${ }^{24}$, then it is an example of an ungrounded, independent and yet extrinsic property.

Another potential example of a fundamental property which can be instantiated in both an intrinsic and an extrinsic fashion is $\operatorname{spin} .{ }^{25}$ In the case of fermions such as electrons, protons, neutrons, neutrinos and quarks, spin is instantiated according to the Pauli Exclusion Principle which states that like fermions cannot be in the same quantum state simultaneously. For instance, a neutral helium-4 atom has two electrons bound in the same orbital, electrons which must obey the Pauli Exclusion Principle and therefore cannot be in the same quantum state as each other: if one electron has spin $1 / 2$, the other must have spin -1/2. Spin, or more specifically having spin $1 / 2$, is a good candidate to be a fundamental property and yet one electron's instantiating having spin $1 / 2$ is dependent upon the existence of the other electron having spin-1/2. If we regard the electrons in the helium-4 atom as distinct individuals, their respective spin values are instantiated in an extrinsic fashion. If spin is fundamental, this provides an example of an extrinsic fundamental property.

Furthermore, one might extend this example because particles do not always obey the Pauli Exclusion Principle. Bosons, such as photons, which have whole integer spin never do so.

Furthermore, although fermions always obey the principle, it does not apply to fermions which are not in the appropriate pairs or groups: for instance, the spin of an individual, isolated, unentangled electron is instantiated independently of whether the electron is lonely or accompanied, having spin $1 / 2$ (say) can be instantiated in an intrinsic fashion. This phenomenon would make spin a locally intrinsic property and thus one able to satisfy Langton and Lewis's four conditions of independence. Having spin 1/2 is an ungrounded, independent and yet extrinsic property.

Examples such as these suggest that despite the intuitive plausibility of presupposing that all ungrounded independent properties are intrinsic, there is a genuine possibility of independent,

24 Pace Bauer.

25 It is worth noting that in physics spin is sometimes described as an intrinsic property, more specifically as intrinsic angular momentum. However, this use of 'intrinsic' is not same as the one at issue in this paper, since in physics 'intrinsic' is being used to distinguish particle spin from whatever 'extrinsic' orbital angular momentum the particle may have. The employment of the term 'intrinsic' in physics does not guarantee that spin is intrinsic in the sense we require. 
ungrounded and yet extrinsic properties; that is, there could be fundamental properties which some particulars have in an intrinsic fashion and others have in an extrinsic fashion.

On the other hand, one might cry foul at such examples: first, because they are notoriously difficult to interpret, especially those concerning the instantiation of quantum properties which obey the Pauli Exclusion Principle, ${ }^{26}$ and second, because one might argue that the examples actually tell us something interesting about the constitutive relationship between grounding and intrinsicality which supports WBT's general strategy.

On the first point, I would stress that the controversy surrounding the interpretation of the examples should not rule them out, since they are merely intended to be an intuitive nudge to counteract the unreflective acceptance of assumption (1) or the more specific presupposition which implies (1) that all fundamental properties are intrinsic. In the current discussion, all that is required of the examples I have given is for it to be possible to interpret them as involving ungrounded properties which are nevertheless only locally intrinsic.

However, to take up the second point, there is a way to interpret the examples of mass and spin which is directly pertinent to the discussion of intrinsicality and grounding, and thus deserves further investigation. In the mass example, for instance, one might suggest that inertial mass is an extrinsic property because a particle has inertial mass in virtue of the Higgs Field; that is, that inertial mass is an extrinsic property because it is partially grounded by the Higgs Field, and thus that this is not an example of an ungrounded extrinsic property at all. Contrary to our usual way of thinking, inertial mass is not a fundamental property. ${ }^{27}$

If one thinks about extrinsic properties as just suggested above - as being instantiated by an individual but partially grounded by something distinct from the individual - then this would give us a good reason to accept assumption (1) that there are no ungrounded, independent and yet extrinsic properties. All locally intrinsic properties would be grounded when they were instantiated in an extrinsic fashion, and so could not be ungrounded in general. ${ }^{28}$ While intrinsic properties could be ungrounded on this view, extrinsic properties could never be ungrounded because extrinsicality requires grounding; and this asymmetry ensures that no properties exist which would satisfy part (a)

26 The example of helium-4 and the Pauli Exclusion Principle raises difficult philosophical problems concerning the individuation of the two electrons in the orbital and one might seek to resolve these difficulties in such a way that my example of spin being locally intrinsic no longer works. For instance, one might interpret the example as showing that quantum particles such as electrons are not self-subsistent individuals (Ladyman and Ross 2007, 13240), and so are not able to have intrinsic properties of their own. To consider this matter in detail would go beyond the scope of this paper, since my argument only requires that there be an interpretation of the example which suggests that spin can be instantiated in an intrinsic or an extrinsic fashion, and thus that (1) might be false.

27 This is the view which Bauer takes, whose example I have borrowed. $(2011,96)$

28 Furthermore, one might not want to allow that a particular property could grounded in some instantiations and ungrounded in others and thus be inclined to say that we have two properties in such cases rather than one. However, I will not pursue this matter here. 
of condition (A) in WBT Grounding and yet turn out to be extrinsic.

The foregoing discussion shows that there is good reason to accept the crucial assumption (1) that there are no independent, ungrounded extrinsic properties, although I have also flagged up some potential reasons to reject it based on properties in current physical theory. The results of the discussion are inconclusive, but one could consistently maintain (1) alongside careful interpretation of the examples of mass and spin which I have given. Hence, the assumption remains plausible and could be explicitly added to WBT Grounding in order to forestall the counterexamples I presented in 3.1 .

What about assumption (2) however? Do we have reason to accept that any property which could be instantiated in an extrinsic fashion (and thus is extrinsic) is at least partially grounded by a property which is not independent? Recall that this assumption is required to deal with the second objection that, according to WBT Grounding, a property P could count as being instantiated by an individual $\mathrm{b}$ in an intrinsic fashion even though $\mathrm{P}$ had at least one grounding property $\mathrm{Q}$ which was instantiated by $\mathrm{b}$ in an extrinsic fashion. All that is required of Q by WBT Grounding is that Q be independent and, as we have seen, extrinsic properties can be independent as long as they are locally intrinsic; that is, if they are instantiated by some individuals in an intrinsic fashion. If the (2) is true however, we have a guarantee that an independent and yet extrinsic Q would itself be at least partially grounded by a property which fails to be independent, and so the counterexamples are eliminated. $^{29}$

Given that (1) is required anyway by WBT Grounding, it will help to clarify the extent to which the truth of (1) constrains whether there are any properties which provide counterexamples to (2): if ungrounded properties cannot be both independent and extrinsic, does this ensure that all extrinsic properties will either be, or be grounded by, a property which is not independent? Unfortunately, it does not, because the possibility remains that there are no fundamental properties and so (1) does not apply; a property Q might be grounded by properties which are instantiated in an extrinsic fashion (and thus would intuitively be instantiated in an extrinsic fashion itself) and yet all of its grounding properties be independent. (2) requires additional support.

Those who are already committed to (1) and to the existence of fundamental properties need not be troubled by this possibility. But it would go against WBT's aversion to ontological commitment to presuppose that these claims hold simply in order to sustain their criterion, and such a move would also restrict the general applicability of WBT Grounding: it would be better to have a criterion of intrinsicality which works whether nature is infinitely complex or not. It seems,

29 For simplicity, I am treating grounding as transitive. However, it would not affect my conclusion were one not to do so. 
therefore, that we require an independent reason for thinking that assumption (2) holds.

The primary source of optimism that one could justify assumption (2) comes from the intuition that there must be a reason for a property's being instantiated in an intrinsic fashion in some individuals and in an extrinsic one others. Since WBT's talk of the different ways in which a property is instantiated was not specific enough to make the distinction clear, one might try to clarify their account further. Perhaps the grounding of the instantiation of a property is different when the properties doing the grounding are themselves intrinsic or are instantiated in an intrinsic manner, compared to when the instantiation of the property is grounded by properties instantiated in an extrinsic fashion. To explicate this difference, one could, perhaps, once again appeal to the connection between grounding and intrinsicality noted in the discussion of assumption (1) which postulated that a property P's being instantiated in an extrinsic fashion essentially involves P's being grounded by entities distinct from the individual which instantiates P. If this is the case, then assumption (2) becomes plausible too: at least one of the properties which ground a property instantiated in an extrinsic fashion will fail to be independent, and so no properties will satisfy condition (A) erroneously. Conversely, building upon a brief suggestion by Rosen $(2010,112)$, one could give a grounding-based account of intrinsicality which utilizes the mereological notion of the instantiation of the properties of an individual being dependent upon the nature of the individual and its parts when those properties are instantiated in an intrinsic fashion. ${ }^{30}$

There would be challenges associated with these projects, however. One would need to explicate what 'the nature of an individual and its parts' involves without presupposing the notion of an intrinsic property (or, relatedly, to clarify what counts as a property instantiated by a distinct particular, without presupposing the intrinsic/extrinsic distinction either). Furthermore, the close connection between intrinsicality and grounding requires that all and only extrinsic properties are externally grounded, but the extrinsicality of some properties might not be due to their instantiation being grounded externally but to some other relation. ${ }^{31}$ A lot turns here on how one should understand grounding, a topic about which I have said very little in this paper.

Furthermore, if one thinks that the most plausible way to underpin assumptions (1) and (2) is by exploiting the relationship between intrinsicality and grounding, perhaps one could avoid the appeal to independence or independence* and use grounding alone to formulate a criterion of intrinsicality instead. A criterion to distinguish intrinsic from extrinsic properties solely in terms of grounding would render Langton and Lewis's independence criterion redundant, making the hybrid

31 See Bauer $(2011,85$ n.10) for some discussion of examples in which the instantiation of extrinsic properties does not appear to require their being grounded by distinct entities. (For instance, an individual's instantiating being $a$ sibling or being a parent may not involve the contemporaneous existence of a living sibling or child respectively, and so we might not want to say that the deceased sibling or child grounds the instantiation of these properties.) 
criterion of WBT Grounding obsolete and with it the assumptions we have been trying to defend, although it would have to avoid the misgivings noted in the previous. Rather than being an amendment of WBT Grounding, this would be an independent alternative to it. It is a proposal which deserves serious consideration, but that will have to wait for another occasion. ${ }^{32}$

\section{Conclusion}

The aim of this paper has been to point out a serious problem concerning the adequacy of WBT Grounding as a criterion of intrinsicality which arises because properties which are intuitively extrinsic are counted as intrinsic according to the criterion. I suggested two strategies to avoid the counterexamples although neither was without problems. The introduction of independence* will make the criterion adequate, but only if independence* can be understood without recourse to a prior understanding of a property's being instantiated in an intrinsic fashion. If it cannot, then an additional assumption is required that there are no ungrounded, locally intrinsic properties.

I gave two examples from fundamental physics which suggest that the ban on locally intrinsic fundamental properties may not be as innocuous as it first appears. This is all the more important since the assumption is also required to maintain the second strategy to save WBT Grounding which makes explicit assumptions about the relationship between properties and grounding in order that the counterexamples do not arise. Both these assumptions are hard to justify, although I suggested that this might be achieved by making a broader appeal to the close relationship between a property's being extrinsic and its being grounded by entities wholly distinct from the individual which instantiates it.

The conclusion of these discussions should, I think, be that WBT Grounding can be defended, but that its position is quite perilous. On one hand, presupposing an outright ban on locally intrinsic fundamental properties involves a level of ontological commitment to the nature of a fundamental category of entities which WBT had hoped to avoid in the formulation of their distinction. Furthermore, the need for such commitment might suggest that Lewis's original appeal to sparse, intrinsic natural properties is not so unpalatable after all, especially since this might be motivated independently from trying to distinguish intrinsic from extrinsic properties, and thus that Lewis's independence criterion could be revived, removing the need to rely upon grounding in the first place. On the other hand, if one chooses to defend WBT Grounding by making a more comprehensive appeal to grounding, the notion of independence seems to become surplus to requirements because one might be able to characterize the criterion in terms of grounding alone.

32 See Marshall 2015 for further discussion. 
Intrinsicality, Independence and Grounding

WBT's hybrid criterion using independence and grounding is under pressure whichever strategy one chooses to defend it.

\section{References}

Bauer, William A. 2011. An argument for the extrinsic grounding of mass. Erkenntnis 74: 81-99.

Bennett, K. 2017. Making things up. Oxford: Oxford University Press.

Chalmers, David J., David Manley, Ryan Wasserman, eds. 2009. Metametaphysics: New Essays on the Foundations of Ontology. Oxford: Oxford University Press.

Correia, F. and B. Schnieder, eds. 2012. Metaphysical Grounding: Understanding the Structure of Reality. Cambridge: Cambridge University Press.

Fine, K. 2012. A Guide to Ground. In Correia and Schnieder, eds. 2012.

Francescotti, Robert M. 1999. How to Define Intrinsic Properties. Noûs 33: 590-609. ed. 2014. A companion to intrinsic properties. Berlin: De Gruyter.

Hale, B. and Hoffmann, A. 2010. Modality: Metaphysics, Logic and Epistemology. Oxford: Oxford University Press.

Harris, R. 2010. How to define extrinsic properties. Axiomathes 20: 461-478.

Hawthorne, J. 2001. Intrinsic Properties and Natural Relations. Philosophy and Phenomenological Research 63: 399-403.

Hoeltje, M., B Schnieder, A. Steinberg, eds. 2013. Varieties of Dependence: Ontological Dependence, Grounding, Supervenience, Response-Dependence. Philosophia Verlag.

Hofweber, T. 2009. Ambitious, Yet Modest, Metaphysics. In Chalmers et al., 2009: 260-289.

Kim, Jaegwon. 1982. Psychophysical Supervenience. Philosophical Studies 51-70. Reprinted in Supervenience and Mind, 1993 (Cambridge: Cambridge University Press): 175-193. 1998. Mind in a Physical World: An Essay on the Mind-Body Problem and Mental Causation. Cambridge, Mass.: MIT Press.

Ladyman, James and Don Ross. 2007. Every Thing Must Go. Oxford: Oxford University Press. Langton, Rae and David Lewis.1998. Defining 'intrinsic'. Philosophy and Phenomenological Research 58: 333-345. Reprinted in David Lewis, Papers in Metaphysics and Epistemology, 1999 (Cambridge: Cambridge University Press): 116-132.

2001. Marshall and Parsons on 'Intrinsic'. Philosophy and Phenomenological Research 63:

* I would like to thank Gonzalo Rodriguez-Pereyra and Alastair Wilder for comments on an earlier draft of this paper. 
$353-5$.

Lewis, D. 1983a. Extrinsic Properties. Philosophical Studies 44: 197-200. 1983b. New Work for a Theory of Universals. Australasian Journal of Philosophy 61: 34377. Reprinted in Mellor and Oliver, eds. (1997): 190-227. 1986a. On the Plurality of Worlds. Oxford: Blackwell. 1986b. Modal Realism at Work: Properties. Abridged section 1.5 from Lewis 1986a, reprinted in Mellor and Oliver, eds. (1997): 173-187.

Marshall, D. 2015. Intrinsicality and Grounding. Philosophy and Phenomenological Research 15: $1-19$.

Marshall, D. and Josh Parsons. 2001. Langton and Lewis on 'Intrinsic'. Philosophy and Phenomenological Research 63: 347-351.

Mellor, D H. and Oliver, A., eds. 1997. Properties. Oxford: Oxford University Press.

Molnar, G. 2003. Powers: a study in metaphysics. Oxford: Oxford University Press.

Mumford, S. 2006. The ungrounded argument. Synthese 149: 471-489.

Rosen, G. 2010. Metaphysical dependence: grounding and reduction. In Hale and Hoffmann, eds. 2010.

Schaffer, J. 2003. Is there a fundamental level? Nô̂s 37: 498-517. 2009. On what grounds what. In Chalmers et al., eds. 2009: 347-83.

Sider, T. 2001. Maximality and Intrinsic Properties. Philosophy and Phenomenological Research 63: 357-64. 2011. Writing the book of the world. Oxford: Clarendon Press.

Taylor, Barry. 1993. On natural properties in metaphysics. Mind 102: 81-100.

Thompson, Naomi. (Forthcoming.) Irrealism about grounding.

Trogdon, K. 2013. An introduction to grounding. In Hoeltje et al., eds. 2013.

Witmer, D. Gene. 2014. A simple theory of intrinsicality. In Francescotti, ed. 2014: 111-138.

Witmer, D. Gene, William Butchard, Kelly Trogdon. 2005. Intrinsicality without Naturalness. Philosophy and Phenomenological Research 70: 326-350. 